\title{
KONFORME VERHEFTUNG UND DIRICHLETSCHES PRINZIP
}

\author{
ALFRED HUBER
}

In der komplexen Ebene bezeichnen $E$ das Innere, $C$ die Peripherie und $A$ das Äussere (incl. $\infty$ ) des Einheitskreises. Ein Homöomorphismus $\Phi$ von $C$ auf sich

$$
\Phi: e^{i \vartheta} \mapsto e^{i \varphi(\vartheta)},
$$

$0 \leqq \vartheta \leqq 2 \pi, \Phi(1)=1$, sei gegeben. Der Einfachheit halber nehmen wir an, $\Phi$ sei analytisch, so dass eine Differentialgleichung der Form

$$
e^{u\left(e^{i \vartheta}\right)} d \vartheta=e^{-v\left(e^{i \varphi}\right)} d \varphi
$$

$(u, v$ harmonisch auf $C$ ) erfüllt ist. Als Folge des Uniformisierungssatzes gibt es eine (analytische) Jordankurve $\Gamma$, dazu eine konforme Abbildung $f$ von $E$ auf das Innere von $\Gamma$ sowie eine konforme Abbildung $g$ von $A$ auf das Äussere von $\Gamma$ derart, dass

$$
f\left(e^{i \vartheta}\right)=g\left(e^{i \varphi(\vartheta)}\right)
$$

für alle $\vartheta$. (Wir normieren $g(\infty)=\infty$, so dass $\Gamma$ bis auf eine ganze lineare Transformation festgelegt ist.)

Es bezeichne $D$ den linearen Raum der harmonischen Funktionen $h: E \rightarrow \boldsymbol{R}$ mit finiten Dirichletintegral,

$$
\iint_{E}\left(h_{x}^{2}+h_{y}^{2}\right) d x d y<\infty .
$$

Durch Einführung des Skalarproduktes

$$
(f, g)_{D}:=\iint_{E}\left(f_{x} g_{x}+f_{y} g_{y}\right) d x d y
$$

wird $D$ zu einem Hilbertraum. (Dabei sind Funktionen miteinander zu identifizieren, welche sich nur um eine additive Konstante voneinander unterscheiden.)

Definition der Abbildung $S: h \mapsto h^{*}$ : Jeder auf $E \cup C$ harmonischen Funktion $h$ wird eine (ebenfalls auf $E \cup C$ harmonische) Funktion $h^{*}$ zugeordnet. Diese ist dadurch charakterisiert, dass - bei geeigneter Wahl der Konstanten - für alle reellen $\vartheta$ die Gleichung

$$
\tilde{h}^{*}\left(\Phi\left(e^{i \vartheta}\right)\right)+\arg \Phi\left(e^{i \vartheta}\right)=\tilde{h}\left(e^{i \vartheta}\right)+\arg e^{i \vartheta}
$$

erfüllt sein soll. (Die Tilde $(\sim)$ wird hier und im folgenden zur Bezeichnung der konjugiert harmonischen Funktion verwendet.) 
Definition des Funktionals $J: h \mapsto J(h):$ Jeder auf $E \cup C$ harmonischen Funktion $h$ wird die Zahl

$$
J(h):=\iint_{E}\left[\left(u_{x}-h_{x}\right)^{2}+\left(u_{y}-h_{y}\right)^{2}+\left(v_{x}-h_{x}^{*}\right)^{2}+\left(v_{y}-h_{y}^{*}\right)^{2}\right] d x d y
$$

zugeordnet. Hier und im folgenden bezeichnet $u$ (respektive $v$ ) diejenige Funktion aus $D$, welche auf $C$ die Randwerte $u\left(e^{i \vartheta}\right)$ (respektive $v\left(e^{i \varphi}\right)$ ) aus Relation (2) annimmt.

Satz. Die Funktion $w(z)=\log \left|f^{\prime}(z)\right|$ ist dadurch charakterisiert, dass

$$
J(w) \leqq J(h)
$$

für alle auf $E \cup C$ harmonischen Funktionen h. Es gilt dann $w^{*}(z)=-\log \left|g^{\prime}\left(\bar{z}^{-1}\right)\right|$, $z \in E$. (Die Funktionen $w$ und $w^{*}$ sind durch $\Phi$ und die Normierung $g(\infty)=\infty$ eindeutig festgelegt.)

Bemerkungen. 1. Eine andere Extremaleigenschaft solcher Verheftungen wurde 1960 von H. Grunsky [2] entdeckt: die Kurve $\Gamma$ ist so beschaffen, dass eine gewisse (durch $\Phi$ bestimmte) Ladungsverteilung auf ihr zur Gleichgewichtsverteilung wird.

2. Bekanntlich gibt es auch nicht-analytische Verheftungen $\Phi$, welche ,,konform zulässig" sind, d. h. für welche eine Jordankurve $\Gamma$ und zugehörige konforme Abbildungen $f$ und $g$ mit den eingangs erwähnten Eigenschaften existieren. Die Theorie der quasikonformen Abbildungen hat auf eine grosse Klasse solcher Verheftungen geführt: Es gelang O. Lehto und K. I. Virtanen [4, 5] sowie - auf dem Weg über ein Resultat von A. Beurling und L. V. Ahlfors [1] - A. Pfluger [6] zu zeigen, dass alle quasisymmetrischen Verheftungen konform zulässig sind. (Die zugehörige Jordankurve $\Gamma$ ist in diesem Falle ein Quasikreis.) Durch das nachstehende Lemma 3 wird nun eine weitere Methode für den Beweis der konformen Zulässigkeit einer ,genügend regulären“ Verheftung nahegelegt: die Orthogonalprojektion im Hilbertraum.

Es gibt jedoch Homöomorphismen $\Phi$, welche nicht konform zulässig sind. Ein Beispiel eines (sogar absolut stetigen) derartigen Homöomorphismus wurde in [3] konstruiert.

\section{Beweis des Satzes}

Lemma 1. Der (auf $E \cup C$ harmonischen) Funktion $w(z)=\log \left|f^{\prime}(z)\right|$ wird durch die Abbildung $S$ die Funktion $w^{*}(z)=-\log \left|g^{\prime}\left(\bar{z}^{-1}\right)\right|$ zugeordnet.

Beweis von Lemma 1. Sei

$$
\log f^{\prime}(z)=p(z)+i \tilde{p}(z), \log g^{\prime}(z)=q(z)+i \tilde{q}(z) .
$$


Aus Gleichung (3) schliessen wir, dass

$$
f^{\prime}\left(e^{i \vartheta}\right) i e^{i \vartheta} d \vartheta=g^{\prime}\left(e^{i \varphi}\right) i e^{i \varphi} d \varphi,
$$

und somit

$$
\begin{gathered}
\exp \left\{p\left(e^{i \vartheta}\right)+i\left[\tilde{p}\left(e^{i \vartheta}\right)+\arg e^{i \vartheta}+\frac{\pi}{2}\right]\right\} d \vartheta \\
=\exp \left\{q\left(\Phi\left(e^{i \vartheta}\right)\right)+i\left[\tilde{q}\left(\Phi\left(e^{i \vartheta}\right)\right)+\arg \Phi\left(e^{i \vartheta}\right)+\frac{\pi}{2}\right]\right\} d \varphi
\end{gathered}
$$

Ein Vergleich von Bedingung (4) mit den Imaginärteilen in den Exponenten der letzten Beziehung lässt $q$ als Bild von $p$ bei der Abbildung $S$ erscheinen. Nun ist aber noch zu berücksichtigen, dass $q$ auf $A \cup C$ (statt auf $E \cup C$ ) definiert ist. Nach Verpflanzung bei Inversion ist $-q$ diejenige harmonische Funktion, zu wlcher $\tilde{q}$ konjugiert harmonisch ist. Man gelangt so $\mathrm{zu}$

$$
w^{*}(z)=-\log \left|g^{\prime}\left(\bar{z}^{-1}\right)\right|, \quad \text { QED. }
$$

Lemma 2. Für alle $\vartheta$ und zugehörigen $\varphi(\vartheta)$ gilt die Gleichung

$$
u\left(e^{i \vartheta}\right)+v\left(e^{i \varphi}\right)=w\left(e^{i \vartheta}\right)+w^{*}\left(e^{i \varphi}\right) .
$$

Dabei sind $u$ und $v$ in Relation (2), $w$ und $w^{*}$ in Lemma 1 definiert.

Beweis von Lemma 2. Aus (2) entnehmen wir

$$
\frac{d \varphi}{d \vartheta}=\exp \left\{u\left(e^{i \vartheta}\right)+v\left(e^{i \varphi}\right)\right\}
$$

Aus (6) folgt

$$
\frac{d \varphi}{d \vartheta}=\frac{\left|f^{\prime}\left(e^{i \vartheta}\right)\right|}{\left|g^{\prime}\left(e^{i \varphi}\right)\right|}=\exp \left\{\log \left|f^{\prime}\left(e^{i \vartheta}\right)\right|-\log \left|g^{\prime}\left(e^{i \varphi}\right)\right|\right\}
$$

Aus (8) und (9) ergibt sich (7), QED.

Im folgenden arbeiten wir mit dem Hilbertraum $H:=D \oplus D$, dessen Elemente die geordneten Paare $[a, b], a$ und $b$ Elemente aus $D$, sind und in welchem das Skalarprodukt mit

$$
([a, b],[c, d])_{H}:=(a, c)_{D}+(b, d)_{D}
$$

definiert ist. In $H$ betrachten wir die Mengen $L:=\left\{\left[h, h^{*}\right]-\left[w, w^{*}\right] \mid h\right.$ harmonisch auf $E \cup C\}, M:=\left\{\left[h, h^{*}\right] \mid h\right.$ harmonisch auf $\left.E \cup C\right\}$. Ferner bezeichne $\Pi$ die abgeschlossene Hülle von $L, \Sigma$ die abgeschlossene Hülle von $M$. Man verifiziert leicht: $L$ ist eine lineare Mannigfaltigkeit, $\Pi$ ein (abgeschlossener) Unterraum. Folglich ist $\Sigma$ eine Hyperebene in $H$. 
Lemma 3. Der Punkt $\left[w, w^{*}\right]$ ist die Orthogonalprojektion des Punktes $[u, v]$ auf die Hyperebene $\Sigma$.

Beweis von Lemma 3. Wir bemerken zunächst, dass

$$
(p, q)_{D}=\iint_{E}\left(p_{x} q_{x}+p_{y} q_{y}\right) d x d y=\int_{C} p \frac{\partial q}{\partial n}|d z|
$$

für irgend zwei auf $E \cup C$ harmonische Funktionen $p$ und $q$. (Hier bezeichnet $n$ die Richtung der äusseren Normalen zu C.) Somit ist

$$
\begin{gathered}
(u-w, h-w)_{D}=\int_{0}^{2 \pi}\left(u\left(e^{i \vartheta}\right)-w\left(e^{i \vartheta}\right)\right)\left[\frac{\partial h}{\partial n}\left(e^{i \vartheta}\right)-\frac{\partial w}{\partial n}\left(e^{i \vartheta}\right)\right] d \vartheta \\
=\int_{0}^{2 \pi}\left(u\left(e^{i \vartheta}\right)-w\left(e^{i \vartheta}\right)\right)\left[\frac{\partial \tilde{h}}{\partial \vartheta}\left(e^{i \vartheta}\right)-\frac{\partial \tilde{w}}{\partial \vartheta}\left(e^{i \vartheta}\right)\right] d \vartheta
\end{gathered}
$$

Wenden wir nun Lemma 2 und Relation (4) an und gehen wir auf die Integrationsvariable $\varphi$ über, so erhalten wir

$$
\begin{gathered}
\int_{0}^{2 \pi}\left(u\left(e^{i \vartheta}\right)-w\left(e^{i \vartheta}\right)\right)\left[\frac{\partial \tilde{h}}{\partial \vartheta}\left(e^{i \vartheta}\right)-\frac{\partial \tilde{w}}{\partial \vartheta}\left(e^{i \vartheta}\right)\right] d \vartheta \\
=-\int_{0}^{2 \pi}\left(v\left(e^{i \varphi}\right)-w^{*}\left(e^{i \varphi}\right)\right)\left[\frac{\partial \tilde{h}^{*}}{\partial \varphi}\left(e^{i \varphi}\right)-\frac{\partial \tilde{w}^{*}}{\partial \varphi}\left(e^{i \varphi}\right)\right] d \varphi \\
=-\int_{0}^{2 \pi}\left(v\left(e^{i \varphi}\right)-w^{*}\left(e^{i \varphi}\right)\right)\left[\frac{\partial h^{*}}{\partial n}\left(e^{i \varphi}\right)-\frac{\partial w^{*}}{\partial n}\left(e^{i \varphi}\right)\right] d \varphi \\
=-\left(v-w^{*}, h^{*}-w^{*}\right)_{D} .
\end{gathered}
$$

Aus (10) und (11) folgt, dass

$$
\begin{aligned}
& \left([u, v]-\left[w, w^{*}\right],\left[h, h^{*}\right]-\left[w, w^{*}\right]\right)_{H} \\
= & (u-w, h-w)_{D}+\left(v-w^{*}, h^{*}-w^{*}\right)_{D}=0
\end{aligned}
$$

für alle Funktionen $h$, welche auf $E \cup C$ harmonisch sind. Der Vektor $[u, v]-\left[w, w^{*}\right]$ steht also senkrecht auf allen zu $L$ gehörigen Vektoren. Damit ist Lemma 3 bewiesen.

Beweis des Satzes. Aus dem Vorangegangenen geht hervor, dass $\left[w, w^{*}\right]$ der am nächsten bei $[u, v]$ gelegene Punkt von $M$ ist. Es gilt

$$
\begin{aligned}
& J(w)=\|[u, v]-\left[w, w^{*}\right] ! !_{H}^{2} \\
& \leqq\left\|[u, v]-\left[h, h^{*}\right]\right\|_{H}^{2}=J(h)
\end{aligned}
$$

für alle $\left[h, h^{*}\right] \in M$. Gleichheit tritt genau dann ein, wenn $\left[h, h^{*}\right]=\left[w, w^{*}\right]$. Damit ist alles bewiesen. 


\section{Literatur}

[1] BeuRling, A., and L. Ahlfors: The boundary correspondence under quasiconformal mappings. - Acta Math. 96, 1956, 125-142.

[2] Grunsky, H.: Eine Grundaufgabe der Uniformisierungstheorie als Extremalproblem. - Math. Ann. 139, 1960, 204-216.

[3] Huber, A.: Isometrische und konforme Verheftung. - Comment. Math. Helv. 51, 1976, $319-331$.

[4] Lehto, O., and K. I. Virtanen: On the existence of quasiconformal mappings with prescribed complex dilatation. - Ann. Acad. Sci. Fenn. Ser. A I Math. 274, 1960, 1-24.

[5] Lehto, O., und K. I. VIRTANEN: Quasikonforme Abbildungen. - Springer-Verlag, BerlinHeidelberg-New York, 1965.

[6] Pfluger, A.: Ueber die Konstruktion Riemannscher Flächen durch Verheftung. - J. Indian Math. Soc. (N.S.) 24, 1960, 401-412.

Eidgenössische Technische Hochschule

Mathematisches Seminar

CH-8092 Zürich

Schweiz

Eingegangen am 12. März 1984 